\title{
Statically Charged Electret Polymers as Solid, Nonvolatile Gates Encapsulating and Tuning Polymer Thermoelectric Parameters
}

R. M. Ireland, T. Jones, H. Li, H. Jang, J. E. West, and H. E. Katz ${ }^{*}$

Prof. H. E. Katz, R. M. Ireland, T. Jones

Department of Materials Science and Engineering and Department of Chemistry, Johns Hopkins University, 3400 North Charles Street, Baltimore, MD

Prof. J. E. West, Department of Electrical and Computer Engineering, Johns Hopkins University, 3400 North Charles Street, Baltimore, MD

\section{Experimental Procedures}

Microscope slides (Corning Inc.) were cleaned by sonication in deionized water, acetone, and 2propanol for 10 minutes each, and blowing dry with $\mathrm{N}_{2}$. Electrode contacts were pre-deposited by thermally evaporating $\mathrm{Au}$ from alumina crucibles using an Edwards thermal evaporation system (50 nm thick, pressure below $5 \times 10^{-6}$ Torr, deposition rate $0.5 \AA \mathrm{s}^{-1}$ ). Deposition rates and thicknesses were monitored by quartz crystal microbalance.

Polymer and additives are weighed in separate glass vials and made up to $10 \mathrm{mg} / \mathrm{mL}$. The solutions are placed on a hot plate $\left(60-80{ }^{\circ} \mathrm{C}\right)$ to enhance solubility. Solutions of F4TCNQ require additional sonication (10 minutes). The polymer is blended in the desired concentration with solvent and, if used, TCNQ, and vigorously stirred before drop-casting. The final blend is dropped by pipette into 2D wells, which are fabricated by laying a pattern of Novec polymer, on glass substrates having pre-deposited gold electrodes. The result is a square $\mathrm{cm}$ semiconductor film with about 5 micrometer thickness, lying over two gold electrodes having a channel gap $(0.2$ $\mathrm{cm}$ across and $0.5 \mathrm{~cm}$ wide). The solvent is allowed to evaporate overnight while samples are kept in a petri dish. Next, samples are placed in vacuum oven overnight $\left(100{ }^{\circ} \mathrm{C}\right.$ under low vacuum) to remove residual solvent. The films are heated under nitrogen atmosphere in the oven (120 ${ }^{\circ} \mathrm{C}$ for $20-30$ minutes). 
Novec is drop-cast onto the devices, about $0.1 \mathrm{~mL}$ per square centimeter of device area, resulting in Novec thicknesses around 5 microns. The Novec dries in a matter of minutes, and devices are further dried under low vacuum at $60{ }^{\circ} \mathrm{C}$ for a couple hours. According to $3 \mathrm{M}$, the recommended environment for applying Novec coating is $20-27{ }^{\circ} \mathrm{C}$ and $40-70 \%$ relative humidity. Humidity and temperature control help to prevent moisture condensation on the polymer film and to provide a reasonable drying time during processing (at $25{ }^{\circ} \mathrm{C}$, a 50 microns thick wet film of Novec 1700 coating can be dried within 5 seconds). Novec can endure temperatures up to 175 ${ }^{\circ} \mathrm{C}$. In the case of cross-linked polystyrene we first deposit an uncrosslinked polymer from solution by drop-casting or spin-casting, and then anneal the film at $200{ }^{\circ} \mathrm{C}$ for two hours, as required to ensure that all the thermally activated sites can be cross-linked. The surface voltage was measured using an electrostatic voltmeter (Isoprobe 279, Monroe Electronics). The electrostatic voltmeter is a surface DC voltmeter that measures surface potential in the range of \pm 3000 volts without physically contacting the measured surface, featuring high-accuracy $(0.1 \%)$, drift-free measurements that are almost fully independent of probe-to-surface separation.

For Seebeck measurements, numerous 2-probe measurements of $\Delta \mathrm{V}$, typically 300 , were made for each value of $\Delta \mathrm{T}$, with a standard deviation of $1-5 \%(0.01-0.05 \mathrm{mV})$ per data set. These $\Delta \mathrm{V}$ were averaged to eliminate the noise signal induced by the environment and obtain just the consistent steady-state value. Six incremental $\Delta \mathrm{T}$ were imposed on the sample, so the slopes of $\Delta \mathrm{V}$ versus $\Delta \mathrm{T}$ give values of the Seebeck coefficient. The linearity of the data of $\Delta \mathrm{V}$ and $\Delta \mathrm{T}$ was used as a key criterion to ensure valid measurements. The setup is checked using Ni metal ingot or Te thin-films deposited from vapor, for which the values we obtain $(-19.8 \pm 0.8 \mu \mathrm{V} / \mathrm{K}$ for $\mathrm{Ni}$, and $\sim 400 \mu \mathrm{V} / \mathrm{K}$ for $\mathrm{Te}$ ) agree well with those reported. Resistivity measurements were employed using 4-probe measurement method with an Agilent 4155C Semiconductor Parameter 
Analyzer using low-resistance probes (Micromanipulators). In the calculation of resistivity based on measured resistances only the thickness of the conducting polymer was used.
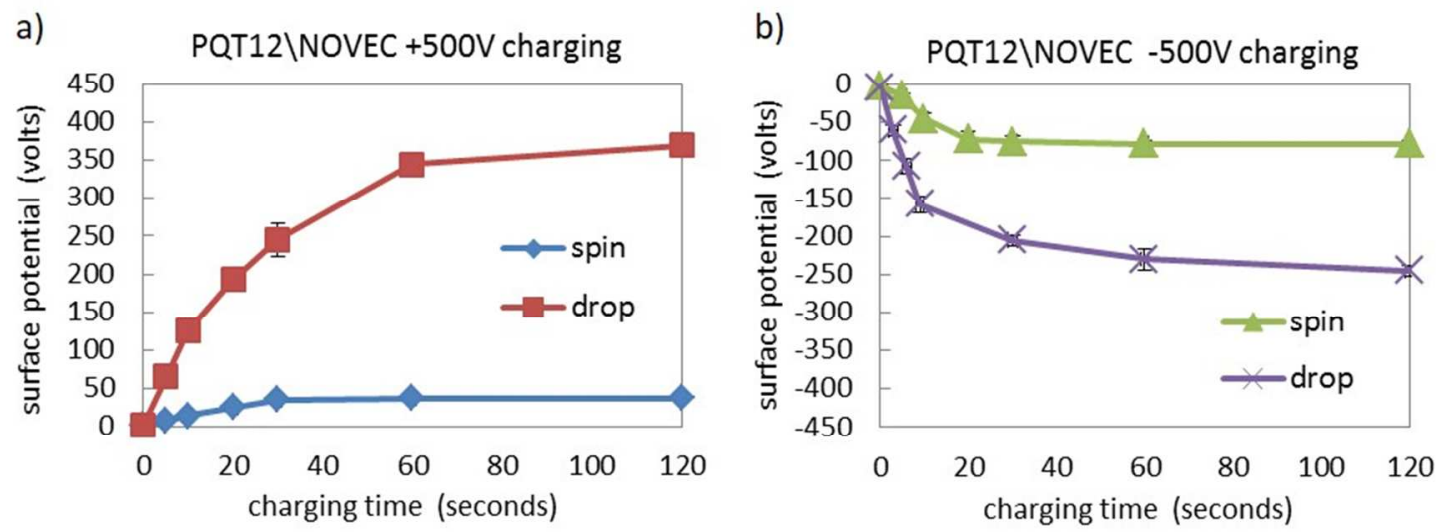

Figure S1. Evolution of surface potential (stored charge) in Novec as a function of charging time at single applied grid voltage of positive or negative $500 \mathrm{~V}$. Spin refers to samples with Novec spin-cast on top, and drop refers to samples with Novec drop-cast on top.

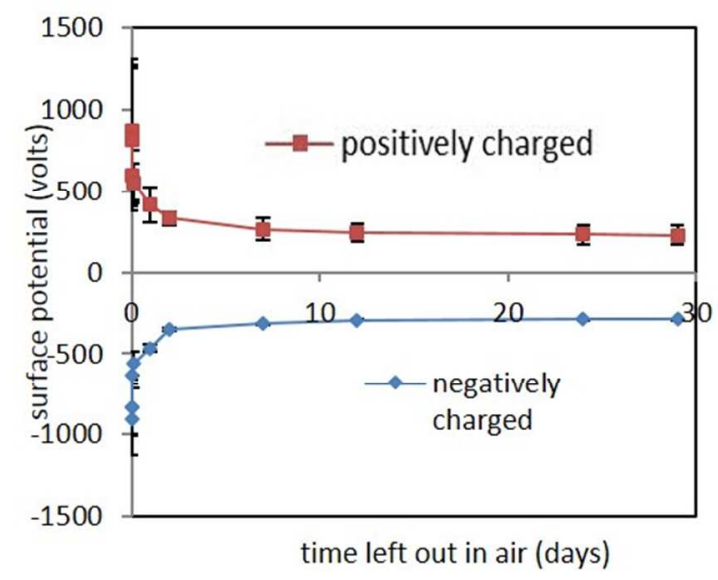

Figure S2. Evolution of surface potential (stored charge) in Novec film deposited over PQT12 as a function of time (days) in air (petri dish) at room temperature (about $20{ }^{\circ} \mathrm{C}$ ). The samples were charged once using grid voltage of $1000 \mathrm{~V}$ applied for 5 minutes, and then all surface potential 
measurements were made over time, and each point is an average of two devices. Points coincident with the y-axis are taken over a few minutes, and represent unstable charge levels.

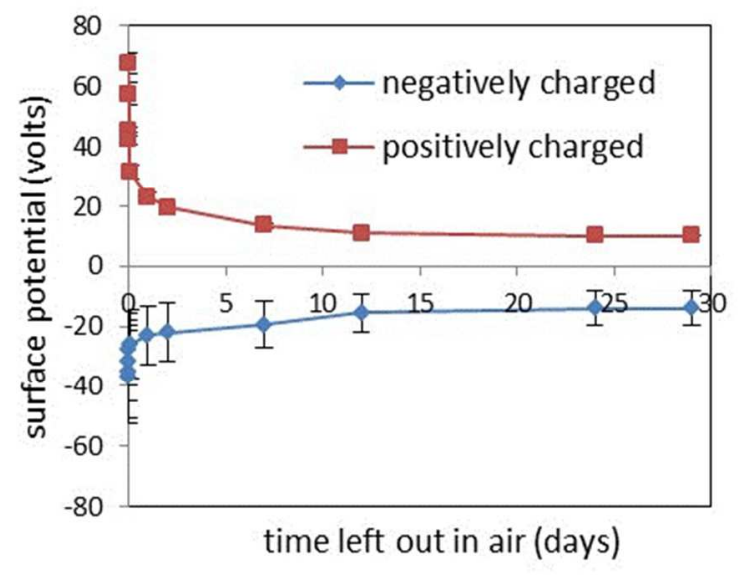

Figure S3. Evolution of surface potential (stored charge) in XLPS film without PQT12 deposited on top, as a function of time (days) in air (petri dish) at room temperature (about 20 ${ }^{\circ} \mathrm{C}$ ). The samples were charged once using grid voltage of $1000 \mathrm{~V}$ applied for 5 minutes, and then all surface potential measurements were made over time, and each point is an average of two devices. Points coincident with the y-axis are taken over a few minutes, and represent unstable charge levels.

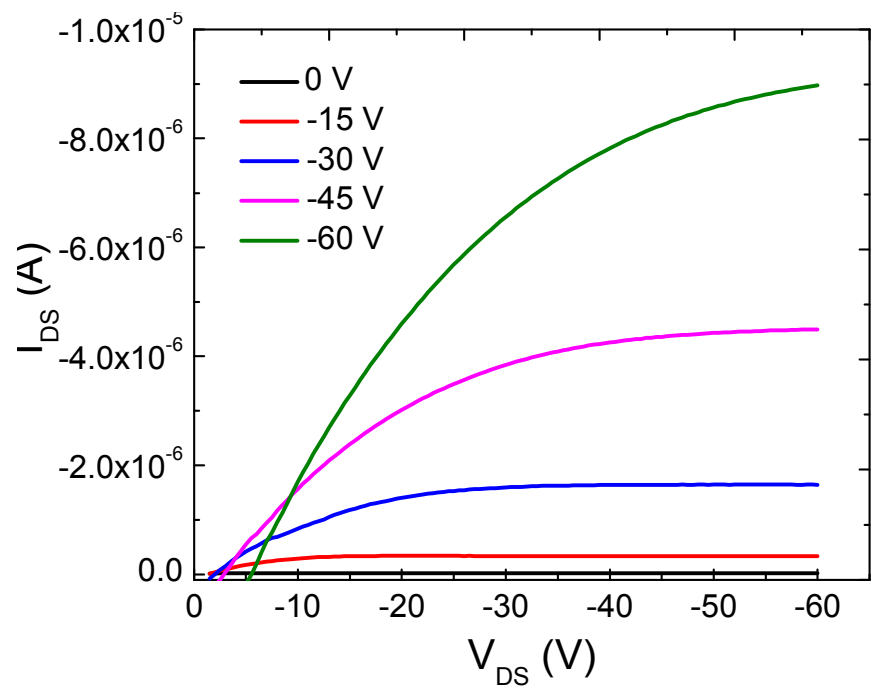


Figure S4. Output curves for a PQT12 transistor on $\mathrm{Si} / \mathrm{SiO}_{2}$ that served as gate and gate dielectric, respectively. The channel dimensions were $8000 \mu \mathrm{m} / 250 \mu \mathrm{m}$, defined by top contact gold electrodes. The $\mathrm{SiO}_{2}$ thickness was $300 \mathrm{~nm}$ and the gate capacitance was $11.5 \mathrm{nF} / \mathrm{cm}^{2}$. The mobility was ca. $0.02 \mathrm{~cm}^{2} / \mathrm{Vs}$.

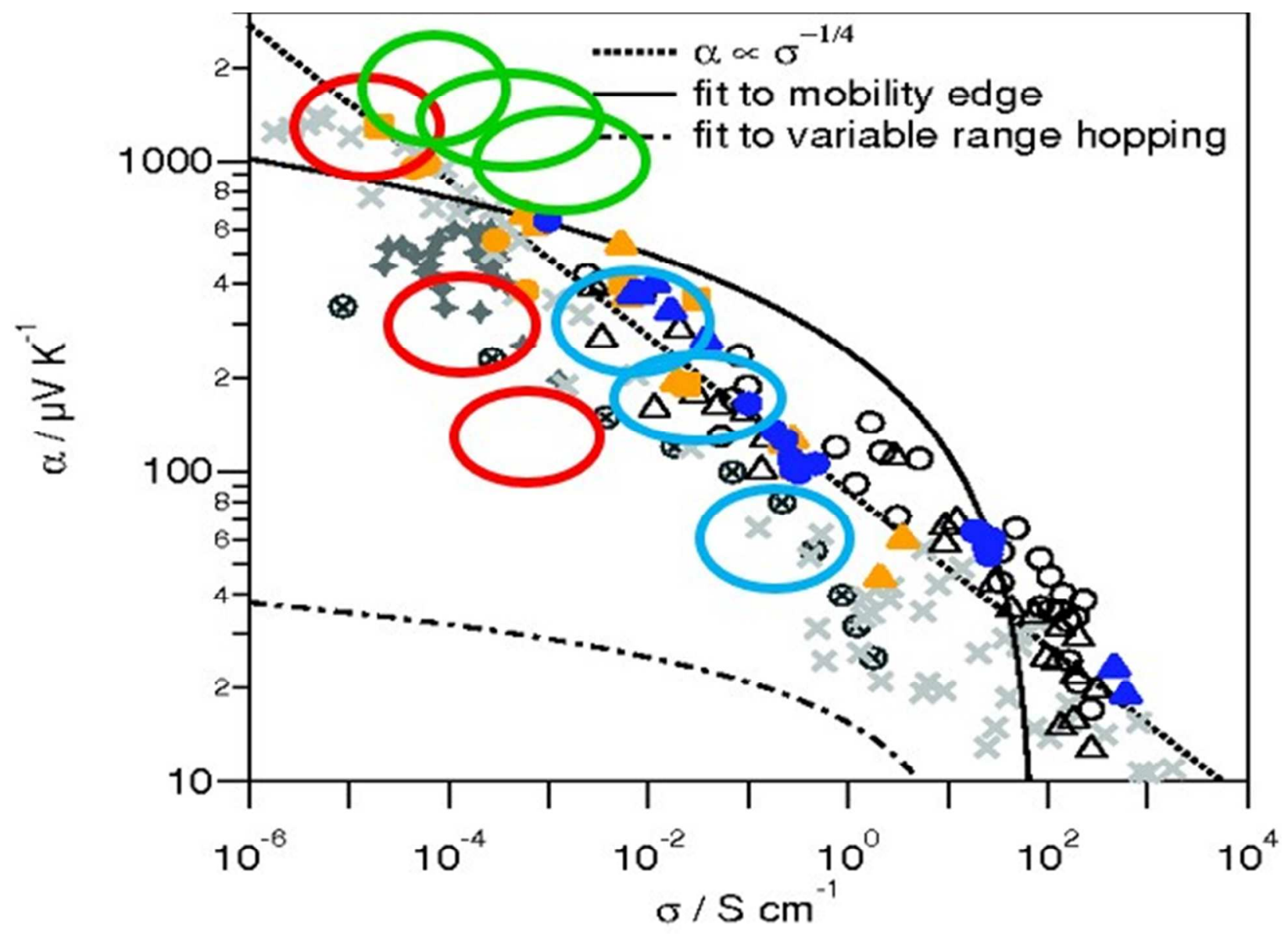

Figure S5. Fits of gated Seebeck data to the Chabinyc empirical model of Reference 1, copyright John Wiley and Sons, used with permission. Oval colors Red: PQT12-Novec; Blue: PQT12-F4TCNQ-Novec; Green, XLPS-PQT12. 